\title{
Emergência e vigor de plântulas de genótipos de feijão-caupi sob estresse salino
}

\author{
Wener S. de Almeida ${ }^{1}$, Francisco R. B. Fernandes ${ }^{2}$, Cândida H. C. de M. Bertini ${ }^{3}$, \\ Marcelo de S. Pinheiro ${ }^{4} \&$ Elizita M. Teófilo ${ }^{3}$
}

\begin{abstract}
RESU MO
Objetivou-se, com este trabalho, avaliar o efeito da salinidade da água de irrigação no feijão-caupi e identificar genótipos tolerantes ao estresse salino nos estádios iniciais do seu desenvolvimento. Os níveis de condutividade elétrica da água foram: $0 ; 2,5 ; 5,0$ e 7,5 dS $\mathrm{m}^{-1}$. A tolerância do feijão-caupi foi avaliada através da redução relativa de matéria seca da parte aérea de cada genótipo. Os resultados foram comparados e analisados pelo teste de Scott-Knott, a 0,05 de probabilidade. Todas as variáveis foram afetadas pelo aumento da salinidade da água de irrigação. 0 genótipo CE-182 mostrou-se mais tolerante nos níveis 2,5, 5,0 e 7,5 dS $\mathrm{m}^{-1}$ na água salinizada com solução de $\mathrm{NaCl}$. No caso da solução salinizada pela mistura dos sais, os genótipos CE-9 e CE-551 foram mais tolerantes.
\end{abstract}

Palavras-chave: Vigna unguiculata, salinidade, tolerância

\section{Emergence and seedling vigor of cowpea genotypes under salt stress}

\begin{abstract}
A B ST RAC T
The objective of this study was to evaluate the effect of salinity of irrigation water on cow pea and identify genotypes tolerant to salt stress in the early stages of its development. The levels of electrical conductivity (EC) of irrigation water were $0,2.5,5.0$ and $7.5 \mathrm{dS} \mathrm{m}^{-1}$. The tolerance of cowpea was evaluated by the relative reduction in shoot dry matter of each genotype. The results were compared and analyzed by Scott-Knott test at 0.05 level of probability. All variables were affected by increasing salinity of irrigation water. The genotype CE-182 was more tolerant at EC of 2.5, 5.0 and $7.5 \mathrm{dS} \mathrm{m}^{-1}$ of water salinized with $\mathrm{NaCl}$. In the solution containing mixture of salts, the genotypes CE-9 and CE-551 were the most tolerant.
\end{abstract}

Key words: Vigna unguiculata, salinity, tolerance

\footnotetext{
${ }^{1}$ Programa de Pós Graduação em Fitotecnia, Departamento de Fitotecnia/UFC. Rua Azevedo Bolão, CEP 60165-455, Fortaleza, CE. E-mail: weneragronomia@gmail.com

2 Programa de Pós Graduação em Engenharia Agrícola, Departamento de Engenharia Agrícola/U FC. Rua 116, CEP 60744-550, Fortaleza, CE. E-mail: ronaldoagroufc@gmail.com

${ }^{3}$ Departamento de Fitotecnia/U FC. Bloco 805, 2977, 60356-001, Fortaleza, CE. E-mail: candida@ufc.br

4 Programa de Pós Graduação em Fitotecnia, Departamento de Fitotecnia/U FC, Rua José Abilio, CEP 60541-052, Fortaleza, CE. E-mail: marcelospufc@gmail.com
} 


\section{INTRODUÇÃO}

Com a expansão do feijão-caupi abrem-se novos mercados e perspectivas de comercialização para esta cultura o que exigirá, dos produtores, a aplicação de manejo mais adequado para a garantia de um produto de qualidade e mais competitivo no mercado interno. A irrigação, por sua vez, é uma das tecnologias aplicadas na agricultura que mais têm contribuído para o aumento na produção de alimentos. No entanto, o uso inadequado desta técnica vem provocando a salinização dos solos, sobretudo nas condições ambientais do Nordeste (Murtaza et al., 2006).

Os problemas com salinidade ocorrem, em geral, em regiões áridas, nas quais a precipitação é menor que a evapotranspiração (Santos et al., 2009b); em áreas irrigadas de modo inadequado o uso de água de baixa qualidade e deficiência na drenagem induz ao acúmulo de sais na superfície do solo (Dantas et al., 2005). Nas plantas, a salinização provoca modificações morfológicas, estruturais e metabólicas (Gonela et al., 2006).

Nas sementes a salinidade afeta a porcentagem de germinação e os caracteres ligados ao vigor, afetando o estabelecimento de plântulas (Sivritepe et al., 2003), reduzindo a velocidade de emergência, na uniformidade, na emergência total, no tamanho inicial e no estabelecimento de estande adequado, fatores que podem influenciar na acumulação de matéria seca e, assim, afetar a produtividade (Scheeren et al., 2010). Tais prejuízos são ocasionados sobremaneira pelo aumento da pressão osmótica na solução do solo, reduzindo a disponibilidade de água para as sementes (Barreto et al., 2010).

A redução no vigor das sementes provocada pelo estresse salino, retardando, deste modo, o estabelecimento das plântulas em campo, é devida à redução na mobilização das reservas e indução de distúrbios nas membranas celulares (Dantas et al., 2003). Com isto, as características associadas ao vigor são mais sensíveis à salinidade do que aquelas associadas à germinação (Santos et al., 2009a).

O grau de tolerância do feijão-caupi ao estresse salino varia entre os diferentes genótipos (Dantas et al., 2002). MurilloAmador et al. (2006) verificaram, trabalhando com diferentes genótipos de feijão-caupi, comportamento diferente entre os genótipos, principalmente entre materiais de distintas procedências.

Em relação ao estabelecimento de plântulas, trabalhos desenvolvidos por Murillo-Amador et al. (2001, 2002) com 25 genótipos de feijão-caupi, demonstraram que o incremento da salinidade no solo reduziu a taxa e a porcentagem de emergência das plântulas, efeito este significativamente dependente do genótipo. Os autores afirmaram que, quanto à tolerância à salinidade, a seleção e a classificação de genótipos de feijãocaupi podem ser realizadas com sucesso durante o estágio de estabelecimento das plântulas.

O objetivo deste trabalho foi avaliar o efeito da salinidade no feijão-caupi e identificar genótipos tolerantes ao estresse salino nos estádios iniciais do seu desenvolvimento.

\section{Material E MÉTODOS}

Os experimentos foram conduzidos no período de outubro a novembro de 2009, em casa de vegetação do Departamento de Fitotecnia do Centro de Ciências Agrárias da Universidade Federal do Ceará (CCA/UFC). Inicialmente, foi realizado um pré-ensaio com trinta genótipos de feijão-caupi, dentre os quais foram selecionados os dez genótipos mais tolerantes ao estresse salino a nível de $10 \mathrm{dS} \mathrm{m}{ }^{-1}$, utilizados no presente trabalho.

O delineamento experimental utilizado foi o inteiramente casualizado, com tratamentos dispostos em esquema fatorial 10 x 4 (genótipos x níveis de condutividade elétrica de irrigação, respectivamente) com quatro repetições, cada uma delas representada por seis copos descartáveis de $250 \mathrm{~mL}$, contendo uma semente em cada um. Os genótipos testados pertencem ao Banco de Germoplasma de feijão-caupi (BAGCaupi) do CCA/ UFC e apresentam, quanto ao porte, características, ciclo, cor da flor e da semente, na grande maioria deles, diferentes (Tabela 1). Os níveis de condutividade elétrica da água de irrigação foram os seguintes: $0 ; 2,5 ; 5,0$ e 7,5 $\mathrm{dS} \mathrm{m}^{-1}$.

Tabela 1. Discriminação dos acessos de feijão-caupi, registro no CCA/UFC, nome de origem e principais características de cada genótipo avaliado

\begin{tabular}{|c|c|c|}
\hline Registro & Nome de origem & Principais características \\
\hline CE-09 & Cara suja-1 & $\begin{array}{l}\text { Enramador, precoce, flor violeta, grão } \\
\text { rajado }\end{array}$ \\
\hline CE-11 & Quebra cadeira & $\begin{array}{l}\text { Enramador, semi-precoce, flor } \\
\text { branca, grão branco com preto }\end{array}$ \\
\hline CE-31 & Pitiúba & $\begin{array}{l}\text { Semi-enramador, precoce, flor } \\
\text { violeta, grão marrom }\end{array}$ \\
\hline CE-67 & Rabo de calango & $\begin{array}{l}\text { Semi-enramador, precoce, flor } \\
\text { violeta, grão marrom }\end{array}$ \\
\hline CE-70 & Quarenta dias-1 & $\begin{array}{l}\text { Enramador, semi-precoce, flor } \\
\text { violeta, grão marrom }\end{array}$ \\
\hline CE-88 & Cariré & $\begin{array}{l}\text { Semi-enramador, semi-precoce, flor } \\
\text { branca, grão branco }\end{array}$ \\
\hline CE-104 & 7917-Dixie hee & $\begin{array}{l}\text { Ereto, precoce, flor branca, grão } \\
\text { marrom }\end{array}$ \\
\hline CE-182 & Var. 16 & $\begin{array}{l}\text { Semi-enramador, semi-precoce, flor } \\
\text { branca, grão marrom }\end{array}$ \\
\hline CE-250 & 154 & $\begin{array}{l}\text { Prostrado, tardio, flor violeta, grão } \\
\text { rajado }\end{array}$ \\
\hline CE-551 & S - 107 & $\begin{array}{l}\text { Enramador, precoce, flor branca, grãc } \\
\text { branco com marrom }\end{array}$ \\
\hline
\end{tabular}

Foram conduzidos dois experimentos: no primeiro, obtiveram-se os níveis de salinidade pela adição de $\mathrm{NaCl}$ e, no segundo experimento, eles foram obtidos pela adição da mistura dos sais $\mathrm{NaCl}, \mathrm{CaCl}_{2} \cdot 2 \mathrm{H}_{2} \mathrm{O}$ e $\mathrm{MgCl}_{2} \cdot 6 \mathrm{H}_{2} \mathrm{O}$ na proporção equivalente de 7:2:1 na água de irrigação, mantendo-se as mesmas condutividades elétricas do primeiro experimento. Para o preparo das soluções salinas nos dois experimentos, obedeceu-se à relação entre condutividade elétrica da água de irrigação e sua concentração $\left(\right.$ mmol $\left._{\mathrm{c}} \mathrm{L}^{-1}=\mathrm{CEa} 10\right)$, conforme Rhoades et al. (2000).

O material utilizado como substrato foi areia lavada, considerada inerte. Inicialmente, foi realizada a aplicação das soluções salinas no solo, por vaso, objetivando-se a indução dos níveis de salinidade desde que a umidade do solo ficasse próxima a $75 \%$ da capacidade de campo. As plântulas permane-ceram por um período de oito dias, tempo este necessário para seu estabelecimento.

As características de tolerância/suscetibilidade dos genótipos de feijão-caupi ao estresse salino avaliadas nos dois 
experimentos, foram: o comprimento da parte aérea (CPA), o comprimento da raiz (CR) e o diâmetro de caule (D); a massa de matéria seca de parte aérea (MSPA) e a de raiz (MSR), realizada em estufa de ar forçado a $80^{\circ} \mathrm{C}$ por $24 \mathrm{~h}$; emergência de plântula (E), correspondendo ao número total de plântulas emergidas; índice de velocidade de emergência (IVE), correspondendo ao número de plântulas emergidas diariamente e tempo médio de emergência (TME). Essas avaliações foram realizadas no oitavo dia, tempo necessário para o estabelecimento das plântulas.

Os resultados obtidos para cada variável foram submetidos à análise de variância. De início, foram realizadas análises univariadas (teste F) em todos os caracteres avaliados, considerando-se fixo o efeito do genótipo. As relações matemáticas entre a salinidade da água de irrigação e as variáveis que expressam o crescimento do feijão-caupi, foram analisadas através da regressão polinomial. Observadas as diferenças significativas pelo teste F, fez-se o teste de média de Scott \& Knott (Scott-Knott, 1974) a 0,05 de probabilidade, através do programa computacional Genes (Cruz \& Carneiro, 2006).

Em referência à classificação dos genótipos quanto sua tolerância ao estresse salino, foram observados os cálculos percentuais de desempenho relativo (aumento ou redução), considerando-se $100 \%$ o valor absoluto do tratamento não salinizado.

Obteve-se a redução da produção (RP) em \% através da fórmula proposta por Fageria et al. (2010):

$$
\mathrm{RP}=\frac{\mathrm{PTNS}-\mathrm{PTS}}{\mathrm{PTNS}} 100
$$

em que:

PTNS - produção do tratamento que não recebeu salinização (salinidade natural do substrato)

PTS - produção do tratamento que recebeu salinização (por nível)

Para proceder à classificação dos genótipos de feijão-caupi em relação à tolerância a salinidade, adotaram-se os seguintes intervalos de redução relativa de MSPA: tolerante, de zero a
20\%; moderadamente tolerante, de 21 a $40 \%$; moderadamente suscetível, de 41 a $60 \%$ e suscetível, acima de 60\% (Fageria et al., 2010). Essas perdas percentuais foram utilizadas como indexadores para comparar a tolerância dos diferentes materiais genéticos.

\section{RESULTADOS E DISCUSSÃO}

Foi encontrada resposta significativa ( $\mathrm{p}<0,01)$ em todas as variáveis avaliadas (Tabela 2), de forma que os níveis salinos da água de irrigação exerceram influência na emergência e no estabelecimento de plântulas.

Trabalhando com feijão-caupi, Silva et al. (2009) relataram resultados semelhantes, pois a salinidade afetou todas as variáveis analisadas, tendo influenciado, portanto, o desenvolvimento da cultura em questão.

Obteve-se a curva de regressão polinomial, em que as equações foram escolhidas com base na sua significância e no valor do coeficiente de determinação $\left(\mathrm{R}^{2}\right)$ (Figuras 1 e 2).

As variáveis analisadas foram afetadas pela salinidade da água de irrigação (Figuras 1 e 2). As variáveis CPA, CR, MSPA e MSR (Figuras 1 e 2 A, B, F e G, respectivamente) foram reduzidos com os incrementos da salinidade.

O efeito da salinidade sobre o CPA e CR, segundo Santos et al. (2009b), é devido, provavelmente, à redução no crescimento da planta, isto em razão do comprometimento de funções fisiológicas e bioquímicas. Por outro lado, Lacerda et al. (2006) relatam que a redução na produção de massa seca da parte aérea e na raiz, está associada aos efeitos osmóticos, tóxicos e nutricionais decorrentes do acúmulo de sais na zona radicular da planta.

A este respeito, Murillo-Amador et al. (2006) relatam que, em biomassa, a redução apresenta correlação linear e significativa com o aumento da concentração de $\mathrm{Na}^{+}$e $\mathrm{Cl}^{-}$nas plantas. Niemam (1965) detectou que a presença desses sais suprime o alongamento e a divisão celular na expansão das primeiras folhas trifoliadas do feijoeiro.

Em referência à produção da biomassa em plantas de feijãocaupi, Dantas et al. (2002) relataram que aumentos na salinidade

Tabela 2. Análise de variância das variáveis analisadas: comprimento de parte aérea (CPA, em cm), comprimento de raiz $(C R$, em $c m)$, diâmetro do caule $(D$, em $c m)$, índice de velocidade de emergência (IVE), massa da matéria seca da parte aérea (MSPA, em g), e da raiz (MSR, em g), tempo médio de emergência (TME, em dias) e emergência (E) de dez genóti pos de feijão-caupi submetidos ao estresse salino com $\mathrm{N} \mathrm{aCl}$ e com a mistura dos sais

\begin{tabular}{|c|c|c|c|c|c|c|c|c|}
\hline \multirow{2}{*}{$\mathbf{F V}^{*}$} & \multicolumn{8}{|c|}{ QM da Análise Univariada } \\
\hline & CPA & CR & D & IVE & MSPA & MSR & TME & $\mathbf{E}$ \\
\hline $\begin{array}{l}\text { Genótipos (G) } \\
\text { Salinidade (S) } \\
\text { Regressão }\end{array}$ & $\begin{array}{r}6,2^{* *} \\
99,8^{* *} \\
482,0^{* *}\end{array}$ & $\begin{array}{r}24,9^{* *} \\
305,8^{* *} \\
343,0^{* *}\end{array}$ & $\begin{array}{c}\text { Estre } \\
1,3^{* *} \\
0,7^{* *} \\
12,0^{* *}\end{array}$ & $\begin{array}{r}\text { lino com N } \\
0,2^{* *} \\
1,9^{* *} \\
188,0^{* *}\end{array}$ & $\begin{array}{r}0,01^{* *} \\
0,02^{* *} \\
636,00^{* *}\end{array}$ & $\begin{array}{r}0,02^{* *} \\
0,04^{* *} \\
23,00^{* *}\end{array}$ & $\begin{array}{r}1,5^{* *} \\
18,9^{* *} \\
33,0^{* *}\end{array}$ & $\begin{array}{c}691,6^{*} \\
6773,9^{* *} \\
21,0^{* *}\end{array}$ \\
\hline CV (\%) & 12,78 & 11,75 & 13,54 & 16,61 & 12,32 & 16,63 & 6,34 & 18,00 \\
\hline $\begin{array}{l}\text { Genótipos (G) } \\
\text { Salinidade (S) } \\
\text { Regressão }\end{array}$ & $\begin{array}{r}8,60^{* *} \\
71,90^{* *} \\
206,58^{* *}\end{array}$ & $\begin{array}{r}\text { Estres } \\
74,10^{* *} \\
469,00^{* *} \\
158,08^{* *}\end{array}$ & $\begin{array}{r}\text { ino com } 2 \\
1,03^{* *} \\
0,60^{* *} \\
56,64^{* *}\end{array}$ & $\begin{array}{c}\text { dos sais } \\
0,22^{* *} \\
1,01^{* *} \\
113,86^{* *}\end{array}$ & $\begin{array}{r}\text { I, } \mathrm{CaCl}_{2} \text { e M } \\
0,002^{* *} \\
0,100^{* *} \\
138,260^{* *}\end{array}$ & $\begin{array}{r}0,01^{* *} \\
0,10^{* *} \\
92,87^{* *}\end{array}$ & $\begin{array}{r}1,20^{* *} \\
12,33^{* *} \\
16,67^{* *}\end{array}$ & $\begin{array}{r}441,50^{* *} \\
1969,30^{* *} \\
48,91^{* *}\end{array}$ \\
\hline CV (\%) & 13,4 & 18,32 & 7,90 & 16,73 & 18,86 & 58,87 & 7,16 & 16,23 \\
\hline
\end{tabular}

${ }^{*} \mathrm{FV}$ - Fonte de variação; ${ }^{\text {ns }}$ - não significativo; ** $\mathrm{e} *$ significativo a 0,01 e a 0,05 pelo teste $\mathrm{F}$, res pectivamente 


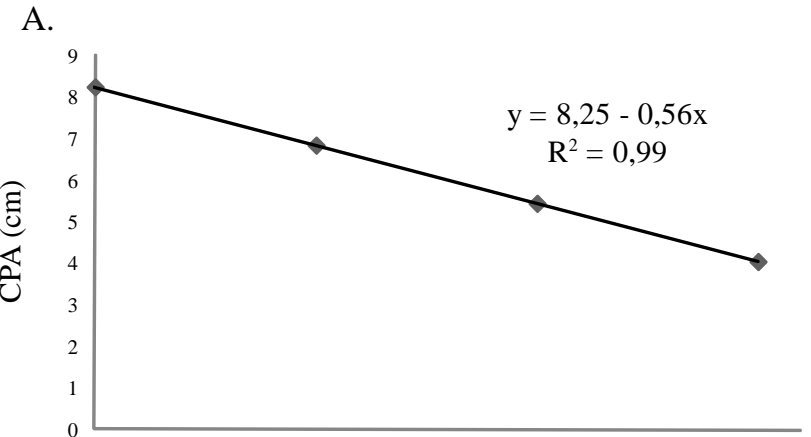

C.

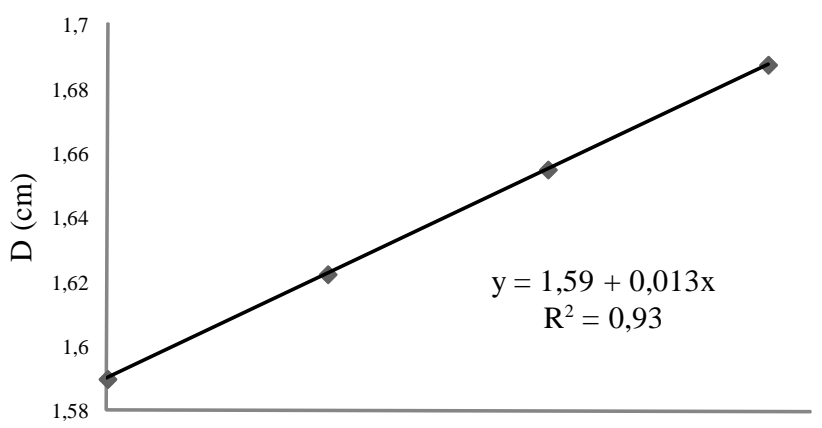

$\mathrm{E}$

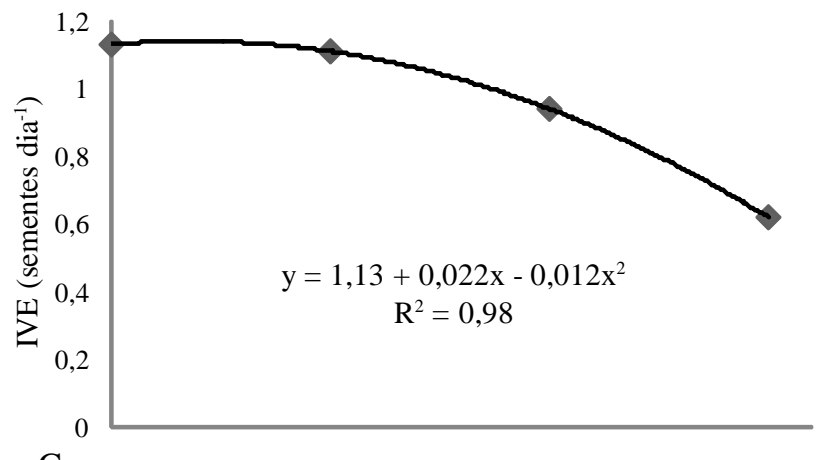

$\mathrm{G}$

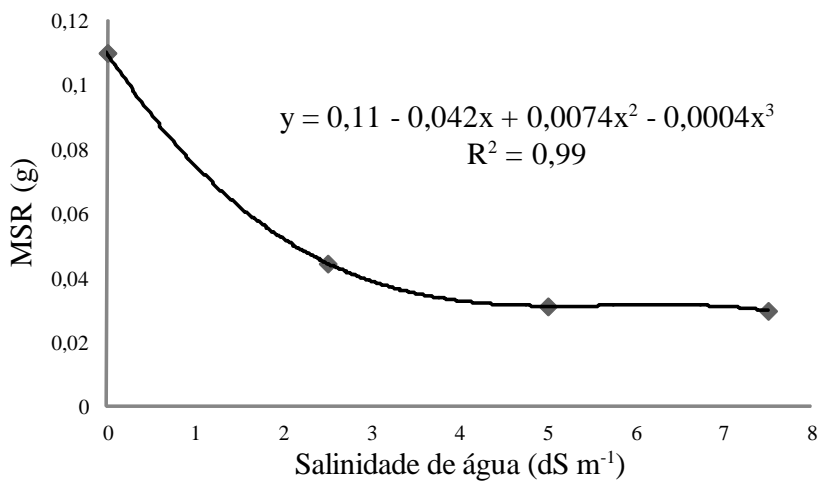

B.

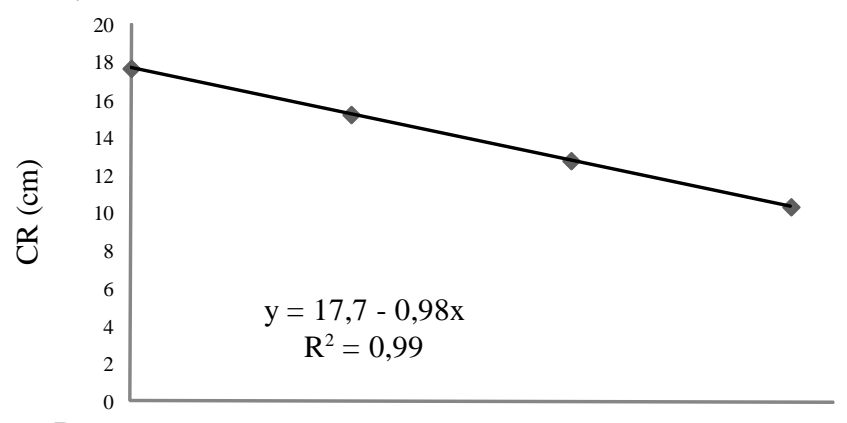

D.

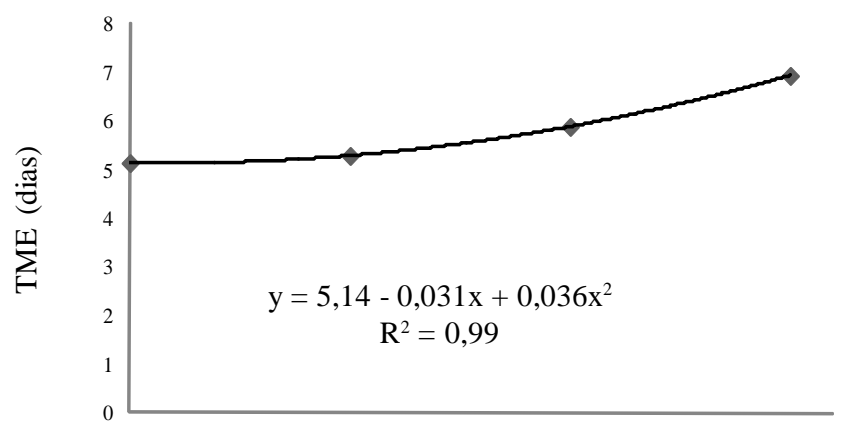

F.

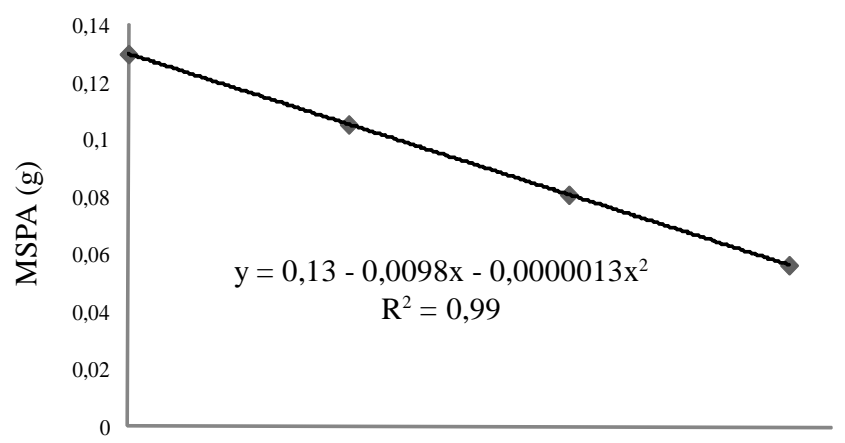

H.

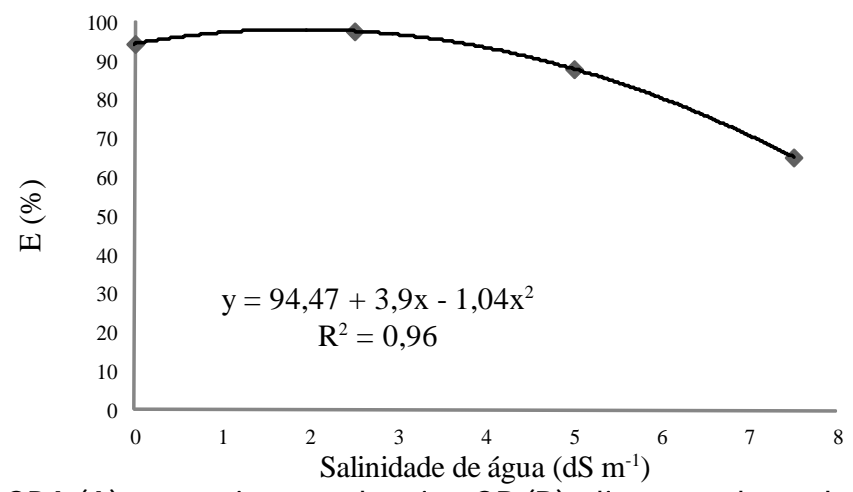

Figura 1. Comportamento de comprimento da parte aérea - CPA (A), comprimento da raiz - CR (B), diâmetro de caule - D (C), tempo médio de emergência - TME (D), índice de velocidade de emergência - IVE (E), matéria de massa seca da parte aérea - MSPA (F), matéria de massa seca da raiz - MSR (G) e emergência - $E(H)$ de dez genótipos de feijão-caupi na fase inicial de desenvolvimento em função do aumento da salinidade provocada pela solução de $\mathrm{NaCl}$

induzem a uma redução de biomassa. Santos et al. (2009a) relatam que em plântulas submetidas a estresse salino o vigor é mais afetado que a germinação, provocando maior redução de biomassa.

Os IVE e E apresentaram redução enquanto o TME sofreu acréscimos (Figuras 1 e 2 E, H e D, respectivamente). Estudos desenvolvidos por Murillo-Amador et al. (2006) demonstraram que o aumento da salinidade diminuiu a percentagem de emergência das plântulas, sendo este efeito significativamente dependente do genótipo.

A germinação das sementes e o estabelecimento das plântulas são afetados, segundo Dantas et al. (2003), em virtude do fato da salinidade reduzir o potencial hídrico da semente em relação ao solo, promovendo atraso da mobilização de enzimas responsáveis pela germinação, além dos efeitos tóxicos dos 
A.
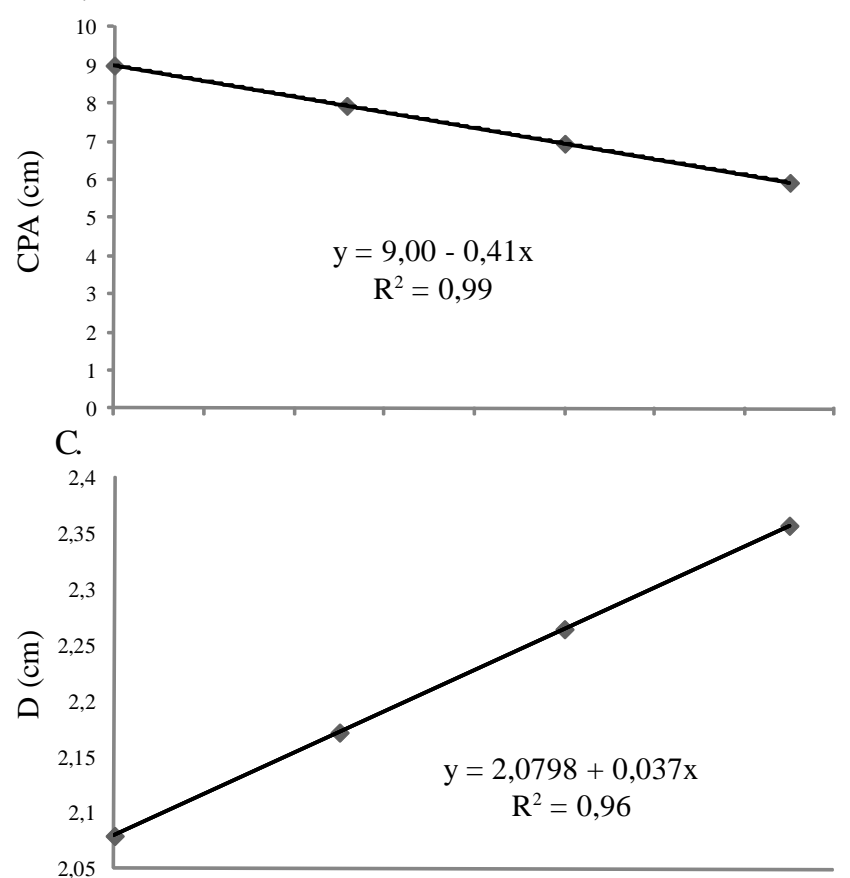

$$
\text { E }
$$
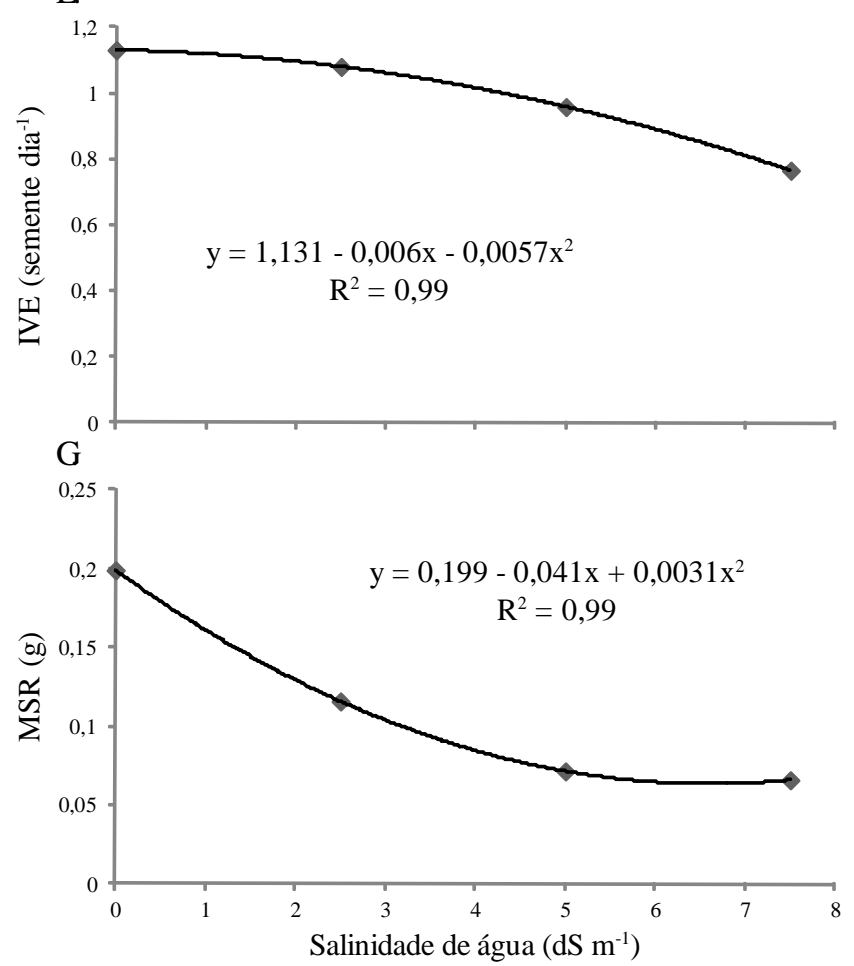
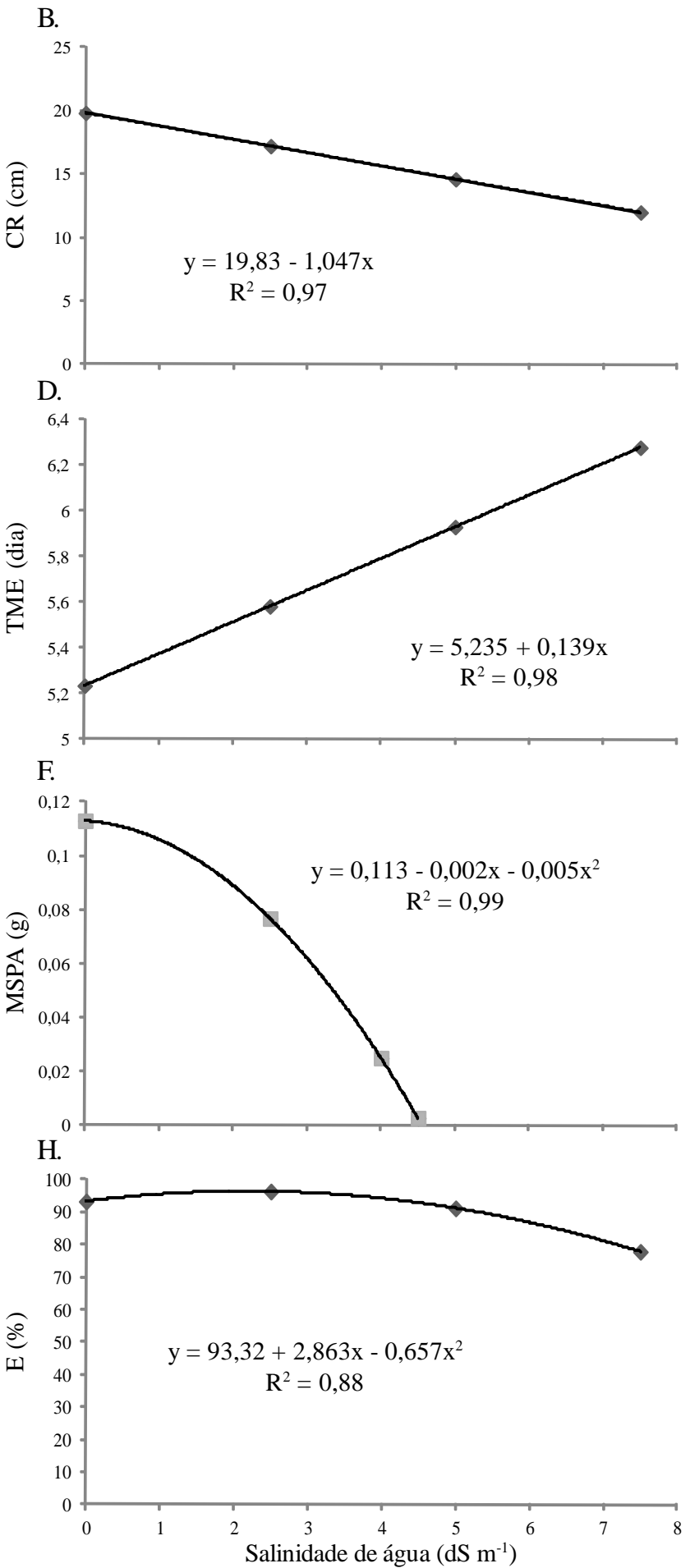

Figura 2. Comportamento de comprimento da parte aérea - CPA (A), comprimento da raiz - CR (B), diâmetro - D (C), tempo médio de emergência - TME (D), índice de velocidade de emergência - IVE (E), matéria da massa seca da parte aérea - MSPA (F), matéria da massa seca da raiz - M SR (G) e emergência - $E(H)$ de dez genótipos de feijão-caupi na fase inicial de desenvolvimento, em função do aumento da salinidade provocada pela mistura dos sais $\mathrm{NaCl}, \mathrm{CaCl}_{2}$ e $\mathrm{M} \mathrm{gCl}_{2}$

sais sobre os tecidos vivos e do retardamento na síntese da enzima $\alpha$-amilase cotiledonar.

O diâmetro da plântula (Figuras 1 e 2 C) apresentou crescimento com o aumento da salinidade. Silva et al. (2009) encontraram, trabalhando com feijão-caupi cv. Quarentinha, resultados diferentes já que, com o aumento da salinidade, o diâmetro do caule reduziu.
Os resultados médios de CPA, CR, D, IVE, MSPA, MSR, TME e E dos dez genótipos de feijão-caupi estudados em função do aumento da salinidade provocada pela solução de $\mathrm{NaCl}$ e pela mistura dos sais $\mathrm{NaCl}, \mathrm{CaCl}_{2}$ e $\mathrm{MgCl}_{2}$, são apresentados na Tabela 3.

Nessa tabela verifica-se o comportamento das variáveis em função dos genótipo, quando submetidos ao estresse 
Tabela 3. M édias de comprimento da parte aérea (CPA), comprimento de raiz (CR), diâmetro (D), índice de velocidade de emergência (IVE), massa seca da parte aérea (MSPA), massa seca da raiz (M SR), tempo médio de emergência (TME) e emergência (E) de genótipos de feijão-caupi na fase inicial de desenvolvimento em função do aumento da salinidade provocada pela solução de $\mathrm{NaCl}$ e pela mistura dos sais $\mathrm{N} \mathrm{aCl}, \mathrm{CaCl}_{2} \mathrm{e} \mathrm{M} \mathrm{gCl}_{2}$

\begin{tabular}{|c|c|c|c|c|c|c|c|c|}
\hline \multirow{2}{*}{ Genótipos } & CPA & CR & D & \multirow{2}{*}{$\begin{array}{c}\text { IVE } \\
\text { semente dia-1 }\end{array}$} & MSPA & MSR & \multirow{2}{*}{$\begin{array}{c}\text { TME } \\
\text { (dias) }\end{array}$} & \multirow{2}{*}{$\begin{array}{c}E \\
(\%)\end{array}$} \\
\hline & \multicolumn{3}{|c|}{$(\mathrm{cm})$} & & \multicolumn{2}{|c|}{ (g) } & & \\
\hline \multicolumn{9}{|c|}{ Estresse salino com NaCl } \\
\hline CE-09 & $6,54 \mathrm{a}$ & $16,79 a$ & $2,60 \mathrm{~b}$ & $1,00 \mathrm{a}$ & $0,08 b$ & $0,06 \mathrm{~b}$ & $5,80 \mathrm{~b}$ & $93,05 a$ \\
\hline CE-11 & $5,60 \mathrm{a}$ & 15,47 a & $2,83 \mathrm{a}$ & $1,08 \mathrm{a}$ & $0,11 \mathrm{a}$ & $0,05 \mathrm{c}$ & $5,43 b$ & $95,83 \mathrm{a}$ \\
\hline CE-31 & $6,13 b$ & 13,87 a & $2,72 \mathrm{a}$ & $0,90 \mathrm{a}$ & $0,09 a$ & $0,08 \mathrm{a}$ & $5,75 b$ & $83,33 a$ \\
\hline CE-67 & $5,63 b$ & $13,54 \mathrm{a}$ & $3,25 \mathrm{a}$ & $0,70 a$ & $0,11 \mathrm{a}$ & $0,06 \mathrm{~b}$ & $6,42 \mathrm{a}$ & $72,22 \mathrm{a}$ \\
\hline CE-70 & $5,19 a$ & 14,18 a & $2,72 \mathrm{a}$ & $0,97 \mathrm{a}$ & $0,08 \mathrm{~b}$ & $0,06 \mathrm{~b}$ & $5,71 b$ & $88,88 \mathrm{a}$ \\
\hline CE-88 & $7,01 \mathrm{a}$ & $14,04 \mathrm{a}$ & $2,92 \mathrm{a}$ & $1,01 \mathrm{a}$ & $0,11 \mathrm{a}$ & $0,05 \mathrm{c}$ & $5,59 \mathrm{~b}$ & $90,28 \mathrm{a}$ \\
\hline CE-104 & 7,39 a & $14,26 \mathrm{a}$ & $3,04 \mathrm{a}$ & 0,87 a & $0,11 \mathrm{a}$ & $7,72 \mathrm{~b}$ & $5,94 \mathrm{~b}$ & $81,94 \mathrm{a}$ \\
\hline CE-182 & $5,87 \mathrm{~b}$ & $14,02 \mathrm{a}$ & $2,50 \mathrm{~b}$ & 0,77 a & $0,09 \mathrm{~b}$ & $0,06 \mathrm{~b}$ & $6,30 \mathrm{a}$ & $76,39 a$ \\
\hline CE-250 & $5,50 \mathrm{~b}$ & $11,90 \mathrm{a}$ & $2,15 b$ & $1,06 \mathrm{a}$ & $0,07 \mathrm{~b}$ & $0,03 d$ & $5,35 b$ & $91,66 \mathrm{a}$ \\
\hline CE-551 & $6,59 a$ & $12,00 \mathrm{a}$ & $2,38 b$ & $0,97 a$ & $0,09 \mathrm{~b}$ & $0,05 \mathrm{c}$ & $5,77 \mathrm{~b}$ & $88,88 \mathrm{a}$ \\
\hline \multicolumn{9}{|c|}{ Estresse salino com a mistura dos sais $\mathrm{NaCl}, \mathrm{CaCl}_{2}$ e $\mathrm{MgCl}_{2}$} \\
\hline CE-09 & $7,81 \mathrm{a}$ & $18,91 \mathrm{a}$ & $2,19 \mathrm{c}$ & $0,99 \mathrm{a}$ & $0,08 \mathrm{~b}$ & $0,10 \mathrm{a}$ & $5,83 \mathrm{~b}$ & $92,71 \mathrm{a}$ \\
\hline CE-11 & $6,41 b$ & $19,36 \mathrm{a}$ & $2,35 b$ & $1,06 \mathrm{a}$ & $0,11 \mathrm{a}$ & $0,11 \mathrm{a}$ & $5,49 \mathrm{~b}$ & $92,71 \mathrm{a}$ \\
\hline CE-31 & $6,95 b$ & $16,04 \mathrm{~b}$ & $2,29 b$ & $0,97 \mathrm{a}$ & $0,08 \mathrm{~b}$ & $0,12 \mathrm{a}$ & $5,81 b$ & $89,58 \mathrm{a}$ \\
\hline CE-67 & $7,76 \mathrm{a}$ & 16,98 a & $2,70 \mathrm{a}$ & $0,79 \mathrm{~b}$ & $0,11 \mathrm{a}$ & $0,11 \mathrm{a}$ & $6,16 \mathrm{a}$ & $79,17 \mathrm{a}$ \\
\hline CE-70 & $6,85 \mathrm{~b}$ & $15,77 \mathrm{~b}$ & $2,29 b$ & $1,05 \mathrm{a}$ & $0,09 \mathrm{a}$ & $0,13 a$ & $5,59 \mathrm{~b}$ & $94,79 a$ \\
\hline CE-88 & $8,60 \mathrm{a}$ & $14,10 \mathrm{~b}$ & $2,07 \mathrm{C}$ & $1,15 \mathrm{a}$ & $0,09 a$ & 0,13 a & $5,43 b$ & 97,91 a \\
\hline CE-104 & $7,86 \mathrm{a}$ & $14,11 \mathrm{~b}$ & $2,42 b$ & $0,85 b$ & $0,10 \mathrm{a}$ & $0,17 \mathrm{a}$ & $6,15 a$ & $85,42 \mathrm{a}$ \\
\hline CE-182 & $7,01 \mathrm{~b}$ & $16,62 \mathrm{a}$ & $2,09 \mathrm{c}$ & $0,85 \mathrm{~b}$ & $0,08 \mathrm{~b}$ & $0,09 a$ & $6,35 a$ & $88,54 \mathrm{a}$ \\
\hline CE-250 & $6,84 \mathrm{~b}$ & $14,54 \mathrm{~b}$ & $1,76 \mathrm{~d}$ & $1,09 \mathrm{a}$ & $0,07 \mathrm{~b}$ & $0,13 a$ & $5,27 \mathrm{~b}$ & $91,66 a$ \\
\hline CE-551 & 8,37 a & $12,64 \mathrm{~b}$ & $2,04 \mathrm{c}$ & $1,02 \mathrm{a}$ & $0,10 \mathrm{a}$ & $0,09 a$ & $5,65 \mathrm{~b}$ & $92,71 \mathrm{a}$ \\
\hline
\end{tabular}

As médias seguidas da mesma letra não diferem estatisticamente entre si pelo teste de Scott-knott, a nível de 0,05 de probabilidade

salino. Os genótipos que apresentaram menor D e TME e maior IVE e E, indicam que as plântulas foram menos afetadas pelo estrese salino; já para CPA, CR, MSPA e MSR, os genótipos que apresentaram os menores valores são os mais afetados.

Houve diferenças nas médias entre os genótipos nas variáveis CPA, D, MSPA, MSR e TME nas soluções de $\mathrm{NaCl}$, porém no CR, IVE e E não ocorreram diferenças significativas entre os genótipos. Para o estresse submetido pela mistura de

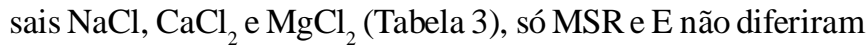
entre os genótipos.

Os genótipos mais afetados pela salinidade da água de irrigação provocada pela solução de $\mathrm{NaCl}$ (Tabela 3 ) em relação ao D, foram: CE-11, CE-31, CE-67, CE-70, CE-88 e CE-104; considerando-se as demais variáveis, os genótipos menos afetados foram: CE-11, CE-31, CE-88 e CE-104.

O genótipo mais afetado pela salinidade da água de irrigação provocada pela mistura dos sais (Tabela 3), em relação ao $\mathrm{D}$, foi o CE-67; tendo em vista as demais variáveis, os genótipos menos afetados pela salinidade foram: CE-11, CE-88 e CE-551.

A tolerância do feijão-caupi à salinidade da água de irrigação provocada pela solução de $\mathrm{NaCl}$ e pela mistura dos sais $\mathrm{NaCl}$, $\mathrm{CaCl}_{2}$ e $\mathrm{MgCl}_{2}$, pode ser avaliada através da Tabela 4.

Na Tabela 4 são apresentados os valores de redução percentual da MSPA dos dez genótipos de feijão-caupi, em cada nível de salinidade, comparativamente à água de irrigação não salinizada. Os danos causados pelo aumento da salinização da água de irrigação são mais expressivos na parte aérea que nas raízes das plantas (Santos et al., 2009b).
Tabela 4. Redução relativa da produção em \% da massa da matéria seca da parte aérea (M SPA) de dez genótipos de feijão-caupi submetidos a quatro níveis de salinidade da água de irrigação

\begin{tabular}{|c|c|c|c|}
\hline \multirow{2}{*}{ Genótipos } & \multicolumn{3}{|c|}{ Níveis de salinidade ( $\mathrm{dS} \mathrm{m}^{-1}$ ) } \\
\hline & 2,5 & 5,0 & 7,5 \\
\hline \multicolumn{4}{|c|}{ Estresse salino com $\mathrm{NaCl}$} \\
\hline CE-09 & $25,42^{\mathrm{MT}}$ & $39,40^{\mathrm{MT}}$ & $54,00^{\mathrm{MS}}$ \\
\hline CE-11 & $22,07^{\mathrm{MT}}$ & $37,88^{\mathrm{MT}}$ & $60,28^{\mathrm{S}}$ \\
\hline CE-31 & $23,11^{\mathrm{MT}}$ & $36,06^{\mathrm{MT}}$ & $57,61^{\mathrm{MS}}$ \\
\hline CE-67 & $10,67^{\top}$ & $31,57^{\mathrm{MT}}$ & $51,96^{\mathrm{MS}}$ \\
\hline CE-70 & $10,58^{\top}$ & $38,51^{\mathrm{MT}}$ & $53,83^{\mathrm{MS}}$ \\
\hline CE-88 & $21,81^{\mathrm{MT}}$ & $51,47^{\mathrm{MS}}$ & $51,32^{\mathrm{MS}}$ \\
\hline CE-104 & $2,65^{\top}$ & $30,16^{\mathrm{MT}}$ & $55,83^{\mathrm{MS}}$ \\
\hline CE-182 & $-4,27^{\top}$ & $20,11^{\mathrm{MT}}$ & $33,94^{\mathrm{MT}}$ \\
\hline CE-250 & $25,83^{\mathrm{MT}}$ & $46,56^{\mathrm{MS}}$ & $62,13^{\mathrm{S}}$ \\
\hline CE-551 & $31,85^{\mathrm{MT}}$ & $37,04^{\mathrm{MT}}$ & $64,13^{5}$ \\
\hline \multicolumn{4}{|c|}{ Estresse salino com a mistura dos sais $\mathrm{NaCl}, \mathrm{CaCl}_{2}$ e $\mathrm{MgCl}_{2}$} \\
\hline CE-09 & $-2,74^{\top}$ & $18,26^{\top}$ & $29,22^{\mathrm{MT}}$ \\
\hline CE-11 & $9,79^{\top}$ & $31,08^{\mathrm{MT}}$ & $39,19^{\mathrm{MT}}$ \\
\hline CE-31 & $13,71^{\top}$ & $22,58^{\mathrm{MT}}$ & $63,71^{\mathrm{s}}$ \\
\hline CE-67 & $-5,65^{\top}$ & $12,37^{\top}$ & $59,36^{\mathrm{MS}}$ \\
\hline CE-70 & $17,09^{\top}$ & $21,09^{\mathrm{MT}}$ & $45,09^{\mathrm{MS}}$ \\
\hline CE-88 & $22,82^{\mathrm{MT}}$ & $21,81^{\mathrm{MT}}$ & $49,66^{\mathrm{MS}}$ \\
\hline CE-104 & $7,32^{\top}$ & $18,68^{\top}$ & $55,31^{\mathrm{MS}}$ \\
\hline CE-182 & $31,17^{\mathrm{MT}}$ & $26,32^{\mathrm{MT}}$ & $47,37^{\mathrm{MS}}$ \\
\hline CE-250 & $-12,57^{\top}$ & $18,86^{\top}$ & $46,86^{\mathrm{MS}}$ \\
\hline CE-551 & $9,50^{\top}$ & $18,25^{\top}$ & $34,22^{\mathrm{MT}}$ \\
\hline
\end{tabular}

Os genótipos são considerados tolerantes ao primeiro nível de salinidade da água de irrigação $\left(2,5 \mathrm{dS} \mathrm{m}^{-1}\right)$ provocada pela solução de $\mathrm{NaCl}$ (Tabela 4), já que as reduções de MSPA não foram superiores a $40 \%$. 
Com a salinidade da água de irrigação a nível de 5,0 $\mathrm{dS} \mathrm{m}^{-1}$. os genótipos não foram classificados como tolerantes, pois todos apresentaram reduções de MSPA superior a $20 \%$.

Os genótipos classificados moderadamente tolerantes a nível de 5,0 dS m ${ }^{-1}$ foram: CE-09, CE-11, CE-31, CE-67, CE-70, CE-104, CE-182 e CE-551; os suscetíveis são: CE-88 e CE-250. A nível de $7,5 \mathrm{dS} \mathrm{m}^{-1}$ o genótipo moderadamente tolerante foi o CE-182; os moderadamente suscetíveis foram: CE-09, CE-31, CE-67, CE-70, CE-88 e CE-104 e os suscetíveis são: CE-11, CE-250 e CE-551.

Com base na salinidade da água de irrigação provocada pela mistura dos sais $\mathrm{NaCl}, \mathrm{CaCl}_{2}$ e $\mathrm{MgCl}_{2}$ os genótipos CE-9, CE-11, CE-31, CE-67, CE-70, CE-104, CE-250 e CE-551 foram classificados tolerantes, a nível de $2,5 \mathrm{dS} \mathrm{m}^{-1}$ (Tabela 4); já o CE-88 e CE-182 foram moderadamente tolerantes.

Para a salinidade ao nível de $5,0 \mathrm{dS} \mathrm{m}^{-1}$ os genótipos tolerantes foram: CE-9, CE-67, CE-104, CE-250 e CE-551; os CE-11, CE-31, CE-70, CE-88 e CE-182 foram moderadamente tolerantes.

Para o nível de 7,5 $\mathrm{dS} \mathrm{m}^{-1}$ os moderadamente tolerantes foram: CE-9, CE-11 e CE-551; os genótipos CE-67, CE-70, CE88, CE-104, CE-182 e CE-250 são moderadamente suscetíveis, enquanto o genótipo CE-31 foi classificado como suscetível o nível de 7,5 dS m-1.

Na comparação dos genótipos tolerantes ao estresse salino provocado pela solução de $\mathrm{NaCl}$ com os tolerantes ao estresse induzido pela mistura dos sais $\mathrm{NaCl}, \mathrm{CaCl}_{2}$ e $\mathrm{MgCl}_{2}$ a classificação em tolerante, moderadamente tolerante, moderadamente suscetível e suscetível, diferiram entre si.

A diferença encontrada na classificação, de acordo com a composição iônica da água de irrigação, segundo Ullah et al. (1993) é devida aos antagonismos iônicos em ambientes salinos, usualmente seguidos pelas deficiências nutricionais; portanto, a composição iônica é importante no que diz respeito à tolerância ao estresse salino.

\section{CONCLUSÕES}

1. O comprimento da parte aérea, comprimento da raiz, diâmetro de caule, índice de velocidade de emergência, massa de matéria seca de parte aérea, massa de matéria seca de raiz, tempo médio de emergência e emergência de plântula foram afetados pelo aumento do nível de salinidade da água de irrigação.

2. O aumento do nível de salinidade da água de irrigação reduziu a emergência e afetou o estabelecimento das plântulas de feijão-caupi.

3. O genótipo CE-182 mostrou-se mais tolerante à salinidade da água de irrigação provocada pela solução de $\mathrm{NaCl}$ nos níveis $2,5,5,0$ e $7,5 \mathrm{dS} \mathrm{m}^{-1}$.

4. Os genótipos CE-9 e CE-551 são tolerantes à salinidade da água de irrigação provocada pela mistura de sais $\mathrm{NaCl}, \mathrm{CaCl}_{2}$ e $\mathrm{MgCl}_{2}$.

\section{Agradecimentos}

Os autores agradecem à Secretaria de Educação Superior (MEC-SeSu) e ao Conselho Nacional de Desenvolvimento Científico e Tecnológico $(\mathrm{CNPq})$ pelo apoio financeiro a esta pesquisa, através da Universidade Federal do Ceará (UFC), pela concessão da bolsa de estudo.

\section{LITERATURA CITADA}

Barreto, H. B. F.; Freitas, R. M. O. de; Oliveira, L. A. de. A.; Araujo, J. A. de M.; Costa, E. M. da. Efeito da irrigação com água salina na germinação de sementes de sabiá (Mimosa caesalpiniifolia Benth). Revista Verde de Agroecologia e Desenvolvimento Sustentável, v.5, p.125-130, 2010.

Cruz, C. D.; Carneiro, P. C. S. Modelos biométricos aplicados ao melhoramento genético, v.2, Viçosa: UFV. 2006. 585p.

Dantas, B. F.; Ribeiro, L. S.; Aragão, C. A. Physiological response of cowpea seeds to salinity stress. Revista Brasileira de Sementes, v.27, p.144-148, 2005.

Dantas, J. P.; Ferreira, M. M. M.; Marinho, F. J. L.; Amorim Nunes, M. S. do; Queiroz, M. F. de; Santos, T. A. dos. Efeito do estresse salino sobre a germinação e produção de sementes de caupi. Revista Agropecuária Técnica, v.24, p.119-130, 2003.

Dantas, J. P.; Marinho, F. J. L.; Ferreira, M. M. M.; Amorim, M. S. N.; Andrade, S. I. O.; Salles, A. L. Avaliação de genótipos de caupi sob salinidade. Revista Brasileira de Engenharia Agrícola e Ambiental, v.6, p.425-430, 2002.

Fageria, N. K.; Soares Filho, W. dos S.; Gheyi, H. R. Melhoramento genético vegetal e seleção de cultivares tolerantes à salinidade. In: Gheyi, H. R.; Dias, N. da S.; Lacerda, C. F. de (ed.); Manejo da salinidade na agricultura: Estudos básicos e aplicativos. Fortaleza: INCT Sal, 2010, cap. 13, p.205-218.

Gonela, A.; Rodrigues, T. J. D.; Parteniani, M. L. S.; Lemos, E. G. M. Tolerância salina em quatro variedades botânicas de Stylosanthes guianensis. Científica, v.34, p.99-106, 2006.

Lacerda, C. F.; Assis Júnior, J. O.; Lemos Filho, L. C. A.; Oliveira, T. S.; Guimarães, F. V. A.; Gomes Filho, E.; Prisco, J. T.; Bezerra, M. A. Morpho-physiological responses of cowpea leaves to salt stress. Brazilian Journal Plant Physiology, v.18, p.455-465, 2006.

Murillo-Amador, B.; Lopez-Aguilar, R.; Kaya, C.; LarrinagaMayoral, J.; Fores-Hernandez, A. Comparative effects of $\mathrm{NaCl}$ and polyethylene glycol on germination, emergence and seedling growth of cowpea. Journal of Agronomy and Crop Science, v.188, p.235-247, 2002.

Murillo-Amador, B.; Troyo-Dieguez, E.; Gárcia-Hernandez, J. L.; Lópes-Agular, R.; Ávila-Serrano, N. Y.; Zamora-Salgado, S.; Rueda-Puente, E. O.; Kaya, C. Effect of $\mathrm{NaCl}$ salinity in the genotypic variation of cowpea (Vigna unguiculata) during early vegetative growth. Revista Scientia Horticulturae, v.108, p.423-431, 2006.

Murillo-Amador, B.; Troyo-Dieguez, E.; Lopes-Cortez, A.; Jones, H. G.; Ayala-Chairez, F.; Tinoco-Ojanguren, C. L.; Salt tolerance of cowpea genotypes in the emergence stage. Australian Journal of Experimental Agriculture, v.41, p.8188, 2001.

Murtaza, G.; Ghafoor. A.; Qadir, M. Irrigation and soil management strategies for using saline-sodic water in a cotton-wheat rotation. Agricultural Water Management, v.81, p.98-114, 2006. 
Nieman, R. H. Expansion of bean leaves and its suppression by salinity. Plant Physiology, v.40, p.156-161, 1965.

Rhoades, J. P.; Kandiah, A.; Mashali, A. M. The use saline waters for crop production. Campina Grande: UFPB, 2000. $117 \mathrm{p}$.

Santos, P. R.; Ruiz, H. A.; Neves, J. C. L.; Almeida, E. F.; Freire, M. B. G. S.; Freire, F. J. Germinação, vigor e crescimento de cultivares de feijoeiro em soluções salinas. Revista Brasileira de Engenharia Agrícola e Ambiental, v.13, p.882-889, 2009a.

Santos, P. R.; Ruiz, H. A.; Neves, J. C. L.; Freire, M. B. G.; Freire, F. J. Acúmulo de cátions em dois cultivares de feijoeiro crescidos em soluções salinas. Revista Ceres, v.56, p.666$678,2009 b$.
Scheeren, B. R.; Peske, S. T.; Schuch, L. O. B.; Barros, A. C. A. Qualidade fisiológica e produtividade de sementes de soja. Revista Brasileira de Sementes, v.32, p.35-41, 2010.

Silva, F. E. O.; Maracajá, P. B.; Medeiros, J. F.; Oliveira, F. A.; Oliveira, M. K. T. Desenvolvimento vegetativo de feijão caupi irrigado com água salina em casa de vegetação. Revista Caatinga, v.22, p.156-159, 2009.

Sivritepe, N; Sivritepe, H. O.; Eris, A. The effect of $\mathrm{NaCl}$ priming on salt tolerance in melon seedling grown under saline conditions. Scientae Horticulturae, v.97, p.229-237, 2003.

Ullah, S. M.; Soja, G.; Gerzabek, M. H. Ion uptake, osmoregulation and plant-water relations in faba beans (Vicia faba L.) under salt stress. Die Bodenkultur, v.44, p. 291-301, 1993. 